\title{
A Data Storage and Management Scheme in Cloud Storage Model
}

\author{
Cai Zehua \\ Department of Computer Science and Technology \\ Wuhan University of Technology \\ Wuhan, China \\ gmiuranus@gmail.com
}

\author{
Wang Shunyan, Long Shangyin, Zhou Haitao \\ Department of Computer Science and Technology \\ Wuhan University of Technology \\ Wuhan, China \\ wangsyan@whut.edu.cn
}

\begin{abstract}
The cloud storage is the evolution of the cloud computing system, which is based on data storage and management. This thesis is focused on the discussion of the cloud storage model that is built on the Cloud Storage Access Protocol of SNIA, and puts forward the concept of storage pooling at storage management layer with unified virtualization and dynamic management on physical storage devices. The thesis also compares the typical DFS (Distributed File System) on the data management layer in different data conditions.
\end{abstract}

Keywords-cloud storage; virtualization; storage pool; distributed file system

\section{INTRODUCTION}

In the cloud computing system, the technology of virtualization spans the IT architecture, making all the system virtualized, including servers, storages, networks, applications, and so on. It realized the unified management, along with the monitor and control of all the resources, which increase the flexibility of the whole system and maximize its efficiency as well. [1] At the same time, virtualization is a key technology to solve the issue of unified management of devices and to realize the flexibility of resource scheduling. In the storage management layer, the cloud storage model can integrate all kinds of storage devices of both same and different structures by the virtualized storage pool, and provide the user of the storage space with a unified, transparent, well encapsulated interface.

When the physical storage comes into the unified management, the file system, at the upper data management layer, can call the storage spaces without difference to make it easier for data access or file management. In the cloud storage model, it is the server cluster, grid computing, and distributed storage technology that achieves the goal of data storage and management. Nowadays, DFS such as Isilon System and IBM SONAS GPFS are the typical products based on integrated hardware \& software design while Google's GFS and Hadoop's HDFS are the typical DFS for separated hardware \& software design. [2]

This thesis is a study on the storage management layer and the data management layer from these above two aspects.

\section{CONCEPT OF ClOUd STORAGE AND ITS MODEL}

\section{A. Background of Cloud Storage}

With the development of technology and the spread of the Internet, the explosion of the information has led us into the information-centric era. According to the study of the IDC (Internet Data Center), the total amount of the information increased from 161EB to 988EB, which is more than 6 times, from year 2006 to year 2010, and in the year 2011, they publish a report called Digital Universe Study- "Extracting Value from Chaos", which predicts that the total amount of the information of world will reach 1.8ZB $\left(1 \mathrm{ZB}=1024 \mathrm{~EB}=1024^{7} \mathrm{~B}\right)$ and growth will double in every two years [3].

The law of conservation never applies for information. In the era of Web2.0, the new Internet service model stimulates the users to transfer from information consumers to information producers. The countless social networking and forums will produce so many data just like snowflakes in blizzard scale, which are mainly the unstructured data type. At the same time, as the advancement of industry information and the business processes go deeper, many data, such as e-bills, financial transactions, satellite detection data, and so on, are copied or created everyday throughout the world. On one hand, the information of the world is increasing so rapidly; while on the other hand, the spaces of mass cheap storage devices are not efficiently used. The purpose of the cloud storage is to integrate the vacant spaces to adapt to the storage of the abundance information.

\section{B. Concept of Cloud Storage}

IDC defines cloud computing as a burgeoning ICT (Information Communication Technology) structure and model, which can be used to build or provide applications, platform, infrastructure and other public services. Technically, cloud computing is deeper integration and development of the traditional computer and network technology, including distributed computing, parallel computing, grid computing, virtualization, loading balance, and so on. It splits the whole computing program into several small programs by the internet, send them into the cloud computing system which consists of several servers, and finally send the results to the users.

Cloud storage is a concept that derives and develops from cloud computing. Strictly speaking, cloud storage is a service [4], an integration of several storage devices and servers, 
which provides data access service. Similar with cloud computing, cloud storage, based on the server cluster, grid technology and distributed storage technology and so on, can synergize a lot of different kinds of storage devices via application to offer data storage and access to the users. In one word, the cloud storage system a cloud computing system whose core is data storage and management.

\section{The Cloud Storage Model}

According to Cloud Storage Tutorial published in 2009 by SNIA (Storage Networking Industry Association), the access protocol for cloud storage is as follows:

TABLE I. The Access Protocol For Cloud Storage

\begin{tabular}{|c|c|c|c|c|c|c|c|}
\hline \multirow{3}{*}{$\begin{array}{l}\text { Standards } \\
\text { Based } \\
\text { Interfaces }\end{array}$} & \multicolumn{2}{|c|}{$\begin{array}{l}\text { File \& Block } \\
\text { Strorage }\end{array}$} & \multicolumn{2}{|c|}{ Linux, Window } & $\begin{array}{r}\text { Lint } \\
F \\
\end{array}$ & $\begin{array}{l}\mathrm{IX}(\mathrm{NFS}, \mathrm{CI} \\
\mathrm{S}, \mathrm{FTP})\end{array}$ & $\begin{array}{l}\text { Windows(CIFS } \\
\text {,FTP) }\end{array}$ \\
\hline & \multicolumn{4}{|c|}{ File \& Block Strorage } & \multicolumn{3}{|c|}{$\operatorname{MAC}(\mathrm{FTP})$} \\
\hline & \multicolumn{4}{|c|}{$\begin{array}{l}\text { Web Services Description } \\
\text { Language and API }\end{array}$} & \multicolumn{3}{|c|}{ Internet (SOAP/REST) } \\
\hline $\begin{array}{l}\text { Interfaces } \\
\text { Services }\end{array}$ & $\begin{array}{l}\text { Multi- } \\
\text { tenancy }\end{array}$ & $\begin{array}{r}\text { De } \\
\text { dup } \\
\text { at }\end{array}$ & & $\begin{array}{c}\text { Policy Base } \\
\text { File } \\
\text { Replicatio }\end{array}$ & & $\begin{array}{c}\text { Policy } \\
\text { Based Geo- } \\
\text { location }\end{array}$ & $\begin{array}{c}\text { Policy } \\
\text { Based } \\
\text { Retention }\end{array}$ \\
\hline Technology & \multicolumn{7}{|c|}{ File System } \\
\hline $\begin{array}{l}\text { \&infrastructu } \\
\text { re }\end{array}$ & \multicolumn{7}{|c|}{ Global Network } \\
\hline
\end{tabular}

The following 5-layer structure of cloud storage model is based on the access protocol above:

TABLE II. LAyer Structure of The Cloud Storage Model

\begin{tabular}{|c|c|} 
Application Access Layer & Application Access \\
\hline Standard Interface Layer & $\begin{array}{c}\text { Standards Based } \\
\text { Interfaces }\end{array}$ \\
\hline Data management Layer & Meta data Management \\
\hline $\begin{array}{c}\text { Storage management } \\
\text { Layer }\end{array}$ & $\begin{array}{c}\text { Storage Virtualization } \\
\text { \& Management }\end{array}$ \\
\hline Storage Devices Layer & $\begin{array}{c}\text { Heterogeneous Storage } \\
\text { \& Network }\end{array}$ \\
\hline
\end{tabular}

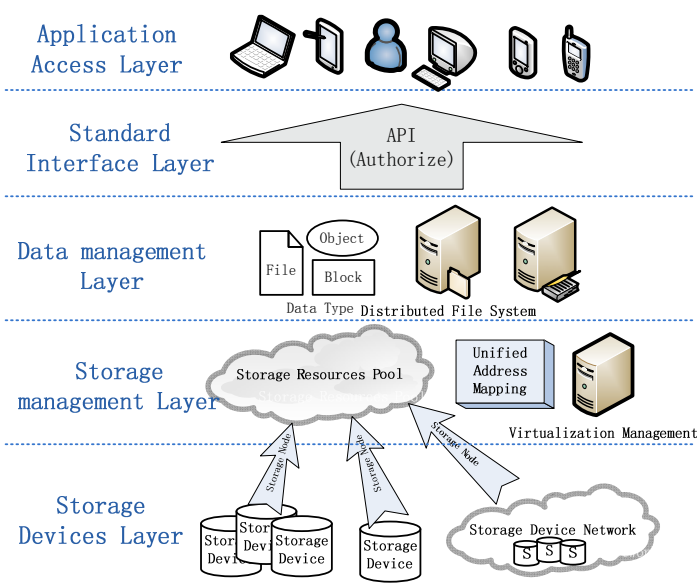

Figure 1. 5-Layer Structure of Cloud Storage Model.

- Storage Devices Layer: Storage Devices Layer consists of storage devices and their networks, such as DAS (Direct Attached Storage), SAN (Storage Area Networking), NAS (Network Attached
Storage), FC SAN, and other heterogeneous devices. They, with a large amount and different distributions, are basic hardware devices for the construction of the cloud storage.

- Storage Management Layer: Storage management Layer, an abstract of the storage devices, simplifies the basic storage structure by integrating all the storage spaces into a storage resources pool, as well as realizes the mapping from physical device to logical view, which we called it virtualization. At this layer, it also makes redundancy management, device condition monitoring, device malfunction maintenance and other functions possible [5].

- Data Management Layer: On the basis of distributed storage technology, data storage on this layer can be divided into block storage, file storage, objected-based storage and table-based storage. We can build proper file systems on these storage types in order to adjust to the keep of structural, semi-structural, and unstructured data and increase the efficiency meaning while $[2,6]$.

- Standard Interface Layer: This Layer is a deeper integration of the three layers listed above which provides a unified interface for the upper applications. Such as the interfaces based on FTP (File Transfer Protocol), HTTP (Hyper Text Transport Protocol), SOAP (Simple Object Access Protocol) and the protocol which used to verify the authorization. The cloud storage service providers can develop diverse interface according to their practical needs.

- Application Access Layer: As its name shows, all kinds of applications are developed at this layer, which guarantee rich cloud storage services for the users.

\section{Design of the Virtualized Storage Pool at the STORAGE MANAGEMENT LAYER}

\section{A. Why Virtualize the Storage?}

Both the cloud computing and the cloud storage actually provide the users with virtualized resource. The SNIA defines storage virtualization as: The act of abstracting, hiding or isolating the internal function of a storage (sub) system or service from applications, compute servers or general network resources for the purpose of enabling application and network independent management of storage or data.

The storage virtualization gathers the storage resources into a huge resource pool to realize the single-point and unified management, during which the change of storage system and the transfer of the data don't need to break off the applications, increasing the dynamic adaptability of the whole system.

\section{B. Storage Pool at the Storage Management Layer}

The cloud storage system of the storage devices layer is usually connected with numbers of physical storage devices of both same and different structure. The dynamic adding or 
moving out of the devices won't influence the normal operation of the whole system. Even if the physical malfunctions happen once in a while, the data can be restored as long as reacting to the system in time. We put forward the concept of storage pooling to realize the unified virtualization and the dynamic management of the physical devices at the bottom of the cloud storage layer, including the design of the table which is used to construct the storage resources pool and the management strategy of these tables.

The pooling of resources is a part of the virtualization, which is widely used in the applications or designs that need to schedule the resources dynamically. Taken the database pool of Tomcat as an example, it can dynamically manage the connection pool to schedule the resources, which is transparent to the upper caller.

It is the same to manage the storage resources in the way of pooling. Each physical storage device or each logical storage device can be regarded as a resource node to be put into the storage pool. In the servers who are in charge of the physical devices at the storage management layer, the storage pool is reflected in the tables. Table 3 includes IDs, types, capacities and running conditions of the devices. When the physical devices are added or deleted, the table will be updated so that the upper can have a clear idea of the condition of the physical storage layer. Servers can use heartbeat detection, namely, periodically testing the effectiveness of every storage node, and it will consume a certain performance to monitor the devices.

TABLE III. LOGICAL MAPPING TABLE

\begin{tabular}{|l|}
\hline DeviceManagement \\
\hline Device_ID \\
\hline Device_Type \\
\hline Device_Capacity \\
\hline Device_Condition \\
\hline$\ldots . .$. \\
\hline
\end{tabular}

If the resources in the storage pool need to be deeper virtualized, that is to say to hide the specific information like the physical addressing or block access, and all of the storage spaces appear in a unified logical address to the upper file system or the data management system, the Device table should be adjusted as following and the physical-logical reflection table, which includes IDs of devices, block sizes, logical indexes, initial address and the max offset, should be added. At the same time, the upper can only see the indiscriminate logical address, and the storage management layer will finish the transfer from logical address to physical address.

\begin{tabular}{|l|}
\hline DeviceManagement \\
\hline Device_ID \\
\hline Device_Type \\
\hline Device_Capacity \\
\hline Device_Condition \\
\hline *Start_Addr \\
\hline *Max_Offset \\
\hline *Block_Size \\
\hline ...... \\
\hline
\end{tabular}

TABLE V. LOGICAL MAPPING TABLE

\begin{tabular}{|l|}
\multicolumn{1}{|c|}{ LogicalMapping } \\
\hline Device_ID \\
\hline Block_Size \\
\hline Logical_Index \\
\hline Start_Addr \\
\hline Max_Offset \\
\hline$\ldots . .$. \\
\hline
\end{tabular}

\section{DFS ChoOsing IN THE DATA MANAGEMENT LAYER}

To make sure of the high viability, reliability and economy, the cloud computing chooses the distributed storage method to storage data and takes the redundancy way to ensure the reliability of the data. [7] The distributed storage technology for now can be divided into block storage, file storage, objected-based storage and table-based storage. [2] This thesis mainly focuses on the comparisons of the applications at the data management layer of the typical DFS-GFS and HDFS.

The real data has characters of size, type, reading/writing frequency, safety and so on. When designing the cloud storage environment, the characters of the data used should be fully taken into consideration, which required to applying the different distributed file systems.

\section{A. Distributed File System-GFS}

GFS (Google File System) is a large DFS based on the cheap servers, which is developed by Google. It regards node failure of server cluster as normal phenomenon and tolerates the faults automatically by software, which guarantees the reliability of system and decrease the cost of the system at the same time.

GFS, as shown in the Figure 2, has one main server, several block servers, and many clients. It achieves lots of design purposes of the traditional distributed file system, such as performances, scalability, reliability and availability. Furthermore, it also has the functions of snapshot and rubbish collection and it guarantees the consistency by data appending.

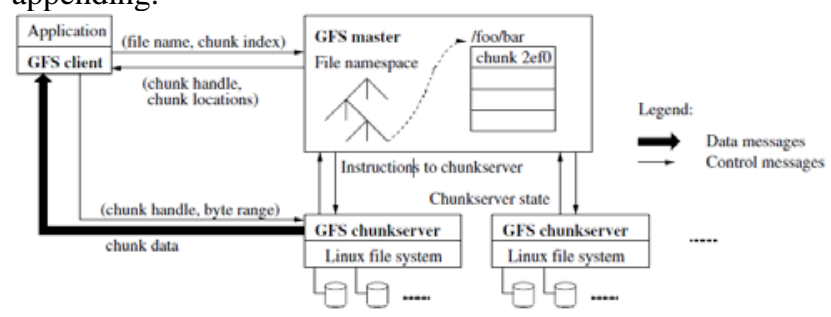

Figure 2. The Structure of GFS [8] 
In GFS, the files will be divided into fixed blocks with a constant 64-bit block handle to identify. To guarantee the reliability, each block will be copied to several block servers with three backups defaulted. The main server is in charge of all the metadata of the management file system, including name space, access control information, the mapping information from the file to block, and the current location.

The formal purpose of the design of the GFS is to serve Google internally to store the huge amount of the data Google generates from his service or production, the GFS is developed for the secondary development of the data as well. From the current Google users and their development, the performances of GFS in dealing with the data are positive. Therefore, when the companies having the same requirement of data dealing construct their own cloud storage environments, may try to get closer to GFS by choosing DFS.

\section{B. Distributed File System-HDFS}

HDFS (Hadoop Distributed File System) is a part of Hadoop project by Apache. HDFS is designed to store large files in the way of flowing, which is proper for the data in the unit of hundred-MB, GB, and TB and they are always in write once read many situation. However, it is not suggested for the access of the low delay data, large numbers of small files, and parallel writing files occasions.

HDFS is inspired by MapReduce and GFS. That's why it has much in common with GFS. However, it has its own way of name for the same concepts. Its structure is shown in the Figure 3.

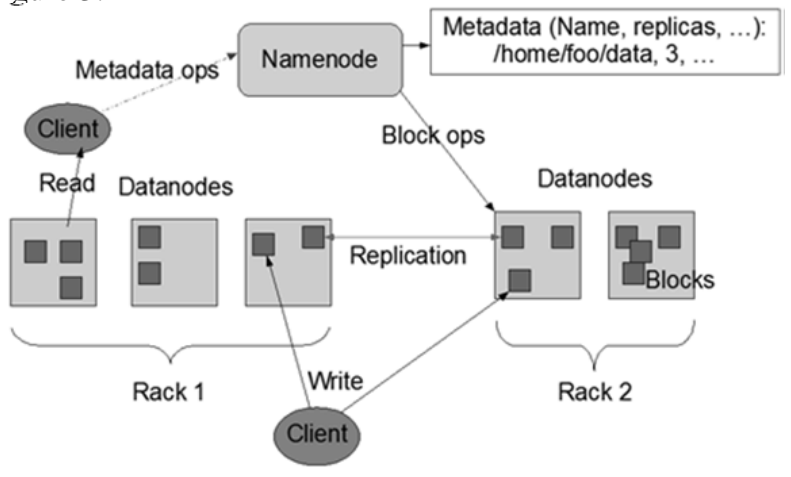

Figure 3. The Structure of HDFS [9]

HDFS defaults basic unit of storage for 64M data block. When a file is smaller than a block unit, it won't take up the whole block data storage space. Compared with the extra functions mentioned in the GFS, HDFS at the moment doesn't have the functions of snapshot, rubbish collection, or the data supplementary.

Although HDFS is weaker than GFS in some functions, HDFS, as an open source frame, should take the different needs of its potential users, such as data-intensive application (like Taobao data storage), compute-intensive application (like Baidu's ranking algorithm), and even mix applications, into consideration at the beginning of its design. Generally, companies can use HDFS in the development of their cloud storage products. The "Cloud Plan" of Yahoo, Intel and other famous IT companies all use HDFS, whose open source is good for the companies to optimize themselves.

\section{CONCLUSION}

The Cloud Storage represents the development of networking storage in the future. Based on the access protocol of the SNIA Cloud Storage, this thesis introduces the five-layer model of cloud storage, and provides some ideas and comparisons on the virtualization and distributed storage technology which are relevant in the storage management layer and data management layer. The thesis especially presents the storage pooling technology used in the cloud storage background to achieve dynamic management and scheduling of storage resource nodes, which is considered to be the basis of the virtualized unification of the logical addresses in storage spaces. The cloud storage technology is now at its beginning stage and those deeper studies and researches on the mature technologies, which are widely applied, in the new environment will make a great contribution to the development and perfection of the cloud storage.

\section{REFERENCES}

[1] De Li, Report of the Cloud Computing Technology Development (2012). Beijing: Science Press,2012.

[2] Liang Liu. Research on Cloud Storage based on Virtualization and Distributed Technology. Anhui: Computer Knowledge and Technology, vol. 8, pp. 2641-2642.2012.

[3] Gantz John,Reinsel David. Digital Universe Study-"Extracting Value from Chaos". 2011. http://idcdocserv.com/1142

[4] Jiyi Wu,Lingdi Ping,Xiaoping Ge,Ya Wang,Jianqing Fu.Cloud Storage as the Infrastructure of Cloud Computing. International Conference on Intelligent Computing and Cognitive Informatics, pp. 380-383. 2010.

[5] Shupeng Li. Research on DFS in the Clould Storage Environment. Beijing:USTC,2011.unpublished.

[6] Guigang Zhang,Chao Li,Yong Zhang,Chunxiao Xing. Research on Cloud Storage Model based on Mass Information Processing. Beijing:Computer Research and Development,vol. 49(suppl), pp.32-36,2012.

[7] Weimin Zhang,Jianfeng Tang,Zhiguo Luo,Ling Qian.Cloud Computing Deeply Change the Future.Beijing:Science Press,2009.

[8] Sanjay,Ghemawat;Howard,Gobioff;Shun-Tak,Leung.The Google File System.http://static.googleusercontent.com/external_content/untruste d_dlcp/research.google.com/zh-CN//archive/gfs-sosp2003.pdf,2003.

[9] Apache.HDFS Architecture Guide. http://hadoop.apache.org /docs/hdfs/ current/hdfs_design.html,2011. 